\title{
Impact of orthognathic surgery on patients' quality of life
}

\begin{abstract}
Objectives: To assess the impact of orthognathic surgery on patients' quality of life.

Participants: 20 patients (males $=10$ ) and (females $=10$ ) with a mean age of 21.4 years who carried out orthognathic surgery with movement of at least one jaw between November 2014 and January 2016.
\end{abstract}

Method: Patients were asked to fill an OHIP-14 questionnaire at two specific intervals, $\mathrm{T}_{1}$ (6 weeks post-surgery) and $\mathrm{T}_{2}\left(6\right.$ months post-surgery). OHIP-14 scores at $\mathrm{T}_{0}, \mathrm{~T}_{1}$ and $\mathrm{T}_{2}$ were compared to assess impact of orthognathic surgery on the quality of life.

Results: There was a significant deterioration in OHIP-14 scores in physiological limitation and social disability domains from base point $\left(\mathrm{T}_{0}\right)$ to $\left(\mathrm{T}_{1}\right)$ 6-weeks.from Base point to 6 months interval $\left(\mathrm{T}_{0}-\mathrm{T}_{2}\right)$ psychological domain improved significantly.

Conclusion: Patient's oral health related quality of life improved significantly following the orthognathic surgery.

\author{
Volume 7 Issue I - 2017
}

\author{
Amna A Al Mutawa,' Emad Eddin M Alzoubi,' \\ Racha Hariri, ${ }^{2}$ Kevin M Mulligan,' Simon \\ Camelleri,' Nikolai J Attard ${ }^{3}$ \\ 'Department of Dental Surgery, University of Malta, Malta \\ ${ }^{2}$ Department of Restorative Dentistry, University of Malta, Malta \\ ${ }^{3}$ Dean of Faculty of Dentistry, University of Malta, Malta
}

Correspondence: Emad Eddin Alzoubi, Department of Dental Surgery, University of Malta, Medical School, Mater Dei Hospital, Block A, Level O, B?Kara Bypass, Msida MSD2090, Malta, Email alzoubiemad@gmail.com

Received: January 30, 2017 | Published: April 17, 2017

\section{Introduction}

Health is a state of complete physical, mental and social well-being and not merely the absence of disease or infirmity (World Health Organization). Orthognathic surgery is the correction of functional and aesthetic abnormalities of the maxilla and mandible. The treatment of such patients involves a combination of orthodontic, surgical and other disciplines. Orthognathic surgery has been indicated for cases where conservative treatment is thought to be inappropriate due to the inherent limitations of orthodontic treatment and so defined as the treatment for dentofacial deformities which includes patients with the following conditions and syndromes. ${ }^{1}$ Patients with significant deformities of the jaw which causes functional and psychological disadvantage-Post-traumatic deformities and malocclusion-Condylar hyperplasia-Hemifacial microstomia-Cleft lip and palate-Obstructive sleep apnoea.

\section{Rationale for orthognathic surgery}

\section{Aesthetics}

Aesthetics is a primary motive for seeking orthognathic surgery from the patient's perspective. One hundred and twenty patients aged 11-33 years, who had undergone orthognathic surgery, were asked to complete a questionnaire to identify their motives for seeking surgery, the degree of satisfaction with surgical results and its effect on their quality of life. It showed that $95 \%$ sought surgery for aesthetics as a primary motive and $85 \%$ were satisfied with surgery outcome and its effect on their quality of life. ${ }^{2}$ A prospective study was carried out on patients scheduled for bilateral sagittal split mandibular osteotomies. These patients were asked to complete a questionnaire to investigate the effect of surgery on the masticatory functions, temporomandibular joint disorders and their satisfaction with the results. Most of these patients $(73 \%)$ reported to be satisfied with the results following the surgical procedure. They reported an improvement in mastication, self-confidence and long-term neurosensory deficits. Patients who underwent mandibular osteotomies with setback were more satisfied with the results than those with mandibular advancement. ${ }^{3}$ When it comes to funds being provided for orthognathic surgery, NHS funding is recommended by priorities committee in order to treat patients with malocclusions and/or jaw with severe skeletal deformity that effect oral function. Patients should be assessed by a multidisciplinary team of specialists and meet the following criteria of having an IOTN of 4 or 5 , quality of life being impacted by functional symptoms, patients having reached skeletal maturation and orthodontics alone is not sufficient to treat functional symptoms. However the priorities committee recommended that NHS funding for orthognathic surgery is placed as low priority, the reason being not enough evidence that there is functional improvement in patients with problems in speech and in patients with jaw pain especially associated with TMJ. However those recommendations do not affect the NHS funding when it comes to orthognathic surgery for treating cleft lip and palate, reconstruction after major surgery or trauma and complex craniofacial surgery. ${ }^{4}$

\section{Psychological aspects}

Psychological assessment for patients seeking orthognathic surgery has been recommended. A structured assessment would allow the clinician to get a clear understanding of the patient concerns as well as their expectations. A thorough evaluation is also necessary to identify patients with body dysmorphic disorder since it was claimed that these patients would not benefit from orthognathic surgery. ${ }^{5}$

\section{Quality of life}

Quality of life is an "individual's perception of their position in life in the context of the culture and value systems in which they live and in relation to their goals, expectations, standards and concerns". Oral health is an important factor that impacts an individual's quality of life. (The World Oral Health Report 2003, Surgeon General's Report on Oral Health). Therefore it is considered an essential part of general and well-being. The term "quality of life" ( QoL ) and "oral health-related quality of life" (OHRQoL) have gained broad recognition over the past five years due to a shift from the traditional clinical dental criteria such as caries or malocclusion towards a more patient-centric oral-health care delivery system that focuses on a 
person's social, emotional and physical experience. Through that they will improve the patient's quality of well-being beyond treatment of only dental disorders. Through OHRQoL the public policy can be advised and help eliminate oral health imbalance. World Health Organisation (WHO) recognizes OHRQoL as an important part of the Global Oral Health Program (2003). ${ }^{6}$ Although malocclusion has a major role in oral health related quality of life. Not all studies show a strong correlation between emotional malocclusion, social well-being and emotional well-being. In 2012 there were more than 2718 orthognathic surgery procedures carried out in England. Orthognathic surgery has demonstrated beneficial effects on the quality of life. The aim of interventions carried out under the NHS are to improve the quality of life..$^{8-12}$ Patients with jaw deformities who and undergo orthognathic surgery are relatively young and receive life-long benefits. Cost-effectiveness of orthognathic surgery has been demonstrated. ${ }^{13}$ Health and effect of health care measurement must include an estimation of well-being and not only an indication of changes in severity and frequency of diseases. This can be carried out by measuring improvement of quality of life related to health care (WHOQOL, 1997). The benefits of orthognathic surgery on quality of life should be considered. Studies have shown that people who are more attractive are considered more favorably and judged to be more sociable, successful and happier than those who are less attractive, the so what is called "what is beautiful is good". ${ }^{14,15}$ There is a complex relationship between one's dento-facial appearance and society's response to it and therefore the desire to change dento-facial appearance is considered more than a superficial wish. Changes in facial/mouth appearance structure and function improves quality of life in the majority of cases, with satisfaction in process and outcome at over $90 \%$ in a great number of audits carried out in UK. Evidence has shown that orthognathic patients at pre-treatment have a quality of life that is poorer than individuals with no dento-facial problems and quality of life related to oral health improves following orthognathic surgery. ${ }^{9-16}$ These finding were supported by a systematic review of the literature and showed that psychological benefit including improved body and facial image, self and social adjustment was experienced by orthognathic patients as an outcome of treatment (Hunt et al.,2001). Improvement in well-being by orthognathic treatment was also noted in another systemic review. ${ }^{17}$ Altered sensation following orthognathic surgery does cause a burden on daily life. A randomized clinical trial showed those two years after surgery patients who had only opening exercises are more likely to report numbness and loss of sensitivity interfering with their daily life than those who received progressive sensory training facial exercises as well as opening exercises. Also older patients and patients who have elevated psychological distress reports are more likely to report altered sensation being persistent and having a negative effect on their daily lives. Therefore clinicians should carefully consider a patients age and psychological well-being while providing counseling prior to surgery on the impact of altered sensation on daily life. ${ }^{18}$

\section{Oral health impact profile- I4}

The Oral Health Impact Profile-14 (OHIP-14) is a short version of the original OHIP-49 questionnaire. It measures the individual's insight of impact of oral condition on their well-being. ${ }^{19}$ OHIP-14 is composed of 14 questions that include 7 domains affecting oral health (functional limitations, physical pain, psychological discomfort, physical disability, psychological disability, social disability and handicap). ${ }^{20,21}$ These 7 domains are subdivided into functional and psychological impact associated with problems including mouth, teeth and orthodontic appliance. The OHIP-14 scale ranges from a frequency of 0 being never to 4 being very often. As a result the final score would fall between a range of 0 , being best condition, no complaints and 56 , being the worst condition, with maximum number of complaints. ${ }^{19}$

Aims: Assess the impact of orthognathic surgery on patients' quality of life.

Materials and method: To prospectively look at a cohort sample of 20 scheduled for orthognathic surgery. They will be asked to fill in the OHIP-14 questionnaire both pre and post-surgery.

Inclusion criteria: 1- Patients who carried out orthognathic surgery with movement of at least one jaw between November 2014 and January 2016.

Exclusion criteria: 1- Patients who had undergone SARPE (surgically assisted rapid palatal expansion) only.

2-Patients who were incapable of completing the questionnaire unaided for any reason.

\section{Method}

a. 20 Patients were asked to fill an OHIP-14 questionnaire at $\mathrm{T}_{0}$ prior to surgery as a baseline.

b. Patients were followed up and asked to fill the OHIP-14 questionnaire at two specified intervals, $T_{1}$ (6 weeks post surgery) and $\mathrm{T}_{2}$ (6 months post surgery).

c. OHIP-14 scores at $\mathrm{T}_{0}, \mathrm{~T}_{1} \& \mathrm{~T}_{2}$ were compared to assess impact of orthognathic surgery on the quality of life.

d. Data collected was entered in an Excel spreadsheet. (Microsoft Corporation-USA).

\section{Statistical analysis}

A Brunner-Langer Anova Type Statistic model was estimated in order to assess differences in OHIP-14 scores over the time.

\section{Results}

Oral Health Related Quality of Life of 20 patients showed that an overall improvement in OHIP-14 scores at $T_{1}$ in comparison to base point $\left(\mathrm{T}_{0}\right)$, following that at $\left(\mathrm{T}_{2}\right)$ the overall OHIP-14 signify a huge improvement in comparison to $\left(\mathrm{T}_{0}\right)$ (Table 1 \& Figure 1). Oral health related quality of life change over time: Each question on OHIP-14 was analyzed individually over periods $\mathrm{T}_{0}, \mathrm{~T}_{1}$ and $\mathrm{T}_{2}$ using the Brunner-Langer ATS test. At $\left(\mathrm{T}_{1}-\mathrm{T}_{0}\right)$ the functional limitation and social disability domains showed a significant deterioration. At $\left(\mathrm{T}_{0}-\right.$ $\mathrm{T}_{2}$ ) interval showed improvement in the psychological discomfort domain in comparison to $\left(\mathrm{T}_{0}-\mathrm{T}_{1}\right)$ (Table 2). It is worth to mention that other domains showed no significant improvement in comparison to base point $\left(\mathrm{T}_{0}\right)$.

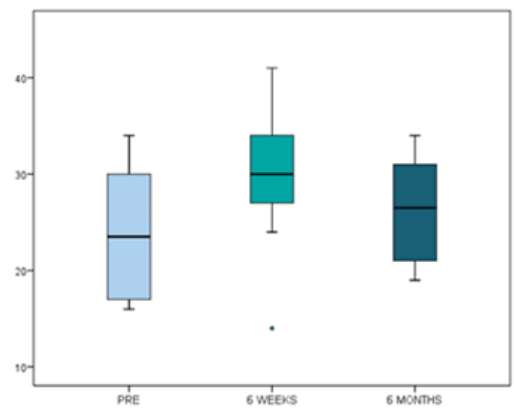

Figure I Distribution of OHIP-I4 score from $\mathrm{T}_{0}$ to $\mathrm{T}_{2}$. 
Table I Overall OHIP-I4 scores

\begin{tabular}{lll}
\hline OHIP-I4 & Mean & SD \\
\hline$T_{0}$ & 23.7 & 6.5 \\
$\left(T_{0}-T_{1}\right)$ & -6.3 & 9 \\
$\left(T_{0}-T_{2}\right)$ & -2.7 & 7.8 \\
\hline
\end{tabular}

Table $2 * \mathrm{p}<0.05 ;{ }^{*} \mathrm{p}<0.01 ; * * * \mathrm{p}<0.001$

\begin{tabular}{|c|c|c|c|}
\hline Domains & & $\mathbf{T}_{0}-\mathbf{T}_{1}$ & $T_{0}-T_{2}$ \\
\hline \multirow{2}{*}{ Functional limitation } & Troubles to pronounce some words & *Deterioration & No significant \\
\hline & Sense of taste worsened & *Deterioration & No significant \\
\hline \multirow{2}{*}{ Physical pain } & Painful aching & No significant & No significant \\
\hline & Uncomfortable to eat some foods & No significant & No significant \\
\hline \multirow{2}{*}{ Psychological discomfort } & Self-conscious & No significant & *Improvement \\
\hline & Tense & No significant & No significant \\
\hline \multirow{2}{*}{ Physical disability } & Unsatisfactory diet & deterioration & No significant \\
\hline & Interruption of meals & No significant & No significant \\
\hline \multirow{2}{*}{ Psychological disability } & Difficult to relax & No significant & No significant \\
\hline & Embarrassment & No significant & No significant \\
\hline \multirow{2}{*}{ Social disability } & Irritability & No significant & No significant \\
\hline & Difficult to do usual job & **Deterioration & No significant \\
\hline \multirow{2}{*}{ Social handicap } & Life in general less satisfying & No significant & No significant \\
\hline & Totally unable to function & No significant & No significant \\
\hline
\end{tabular}

\section{Discussion}

This prospective research act as a pilot study for a larger future research. It investigated the impact of orthognathic surgery on patient's quality of life, the study consisted of 20 patients who were planned to undergo orthognathic surgery, and patients OHRQoL were assessed using OHIP-14. The main obstacle in the path of this pilot study is the scarcity of sample size since Malta is a very small island. The reasons patients seek orthognathic surgery are often related to esthetics or function, and in most cases such reasons are combined. ${ }^{22}$ Considering that the surgical procedure may result in irreversible changes on the patients' face and personality, both specialist and family should not influence the patients' decision. Thus, the final decision to undergo orthognathic surgery should come from the patients, ${ }^{23}$ the results obtained in this research appeared promising in regards to the shortterm hazards and difficulties that patients go through in order to obtain long-term benefits. Researchers have reported that persons going through orthognathic surgery, who are more concerned with esthetics aspects than others, may have psychological issues. Though, the frequency of people with psychological disorders is higher when it comes to plastic surgery. ${ }^{10,24}$ Found deterioration in functional limitation domain score. OHIP-14 scores at 6 weeks are exactly concordant with them. They reported a significant improvement in psychological status; similar finding was seen in this research as the psychological disability improved toward six month post-surgery. ${ }^{10}$ Did not find significant differences at total OHIP-14 score from basal to 6 weeks, but we have detected a significant improvement. Regarding comparison between basal and 6 month's quality of life, ${ }^{25}$ reported a significant improvement in the overall index and all its specific items. Our results suggest that minor improvement were seen as an overall OHIP 14. A conclusion can be drawn in this research that the OHRQoL in patients who underwent orthognathic surgery showed some improvement in specific domains such as psychological discomfort domain in comparison to base point (To). ${ }^{26-30}$

\section{Conclusion}

Oral Health Related Quality of Life measured with OHIP-14 had demonstrated improvement following the orthognathic surgery.

\section{Acknowledgments}

None.

\section{Conflicts of interest}

The authors declare there are no conflicts of interest.

\section{References}

1. BAOMS Interim Clinical Commission Policy: Orthognathic Surgery.

2. Sadek H, Salem G. Psychological aspects of orthognathic surgery and its effect on quality of life in egyptian patients. Eastern Mediterranean health journal. 2007;13(1):150-157.

3. Pahkala RH, Kellokoski JK. Surgical-orthodontic treatment and patient's functional and psychological well-being. American Journal of Orthodontics and Dentofacial orthopaedics. 2005;132:158-163. 
4. HIOW/SHIP. Priorities Committee April 2008 to March 2012 Policy Recommendations. 2014

5. Cunningham SJ, Feinmann C. Psychological assessment of patients requesting orthognathic surgery and the relevance of body dysmorphic disorder. British Journal of Orthodontics. 1998;25(4):293-298.

6. Sischo L, Broder HL. Oral Health-Related Quality of Life: What, Why, How and Future Implications. J Dent Res. 2011;90(11):1264-1270.

7. Commission guide: Orthognathic Procedures. British Association of Oral and Maxillofacial Surgeons, London, England, UK, 2013:1-24.

8. Cunningham SJ, Garratt AM, Hunt NP. Development of a conditionspecific quality of life measure for patients with dentofacial deformity: 1 Reliability of the instrument. Dent Oral Epidemiol. 2000;28(3):195-201.

9. Cunningham SJ, Garratt AM, Hunt NP. Development of a conditionspecific quality of life measure for patients with dent facial deformity: 11. Validity and responsiveness testing. Community Dent Oral Epidemiol. 2002;30(2):81-90.

10. Lee S, Mc Grath C, Samman N. Impact of orthognathic surgery on quality of life. J Oral Maxillofac Surg. 2008;66(6):1194-1199.

11. Criteria for Orthognathic surgery. The American Association of Oral and Maxillofacial Surgeons. 2008

12. Motegi E, Hatch JP, Rugh JD, et al. Health-related quality of life and psychosocial function5 years after orthognathic surgery. Am J Orthod Dentofacial Orthop. 2003;124(2):138-143.

13. Esperao PT, De Oliveira BH, De oliveira Almeida MA, et al. Oral healthrelated quality of life in orthognathic surgery patient. Am J Orthod Dentofacial Orthop. 2010;137(6):790-795.

14. Cunningham SJ, Sculpher M, Sassi F, et al. A cost-utility analysis of patients undergoing orthognathic treatment for the management of dentofacial disharmony. Br J Oral Maxillofac Surg. 2003;41(1):32-35.

15. Dion K, Berschied E, Walster E. What is beautiful is good. J Pers Soc Psychol. 1972;24(3):285-290.

16. Eagly AH, Ashmore RD, Makhijani MG, et al. What is beautiful is good, but...: A meta-analytic review of research on the physical attractiveness stereotype. Psychol Bull.1991;110(1):109-128.

17. Murphy C, Kearns G, Sleeman D, et al. The clinical relevance of orthognathic surgery on quality of life. Int J Oral Maxillofac Surg. 2011;40(9):926-930.
18. Alanko OM, Svedstrom Oristo AL, et al. Patients perception of orthognathic treatment, eel-being, and psychological or psychiatric status: a systemic review. Acta Odontol Scand. 2010;68(5):249-260.

19. Phillips C, Kim SH, Tucker M, et al. Sensory retraining: Burden in daily life related to altered sensation after orthognathic surgery, a randomised clinical trial. Orthod Craniofac Res. 2010;13(3):169-178.

20. Zimmer S, Nora B, Ebtehal G, et al. Association between oral health related and general health-related quality of life in subjects attending dental offices in Germany. J Public Health Dent. 2009;70(2):160-167.

21. Slade GD, Spencer AJ. Social impact of oral conditions among older adults. Australian Dental Journal. 1994;39(6):358-364.

22. Slade GD. Derivation and validation of a short-form oral health impact profile. Community Dent Oral Epidemiol. 1997;25(4):284-290.

23. Bellucci CC, Kapp-Simon KA. Psychological considerations in orthognathic surgery. Clin Plast Surg. 2007;34(3):e11-e16.

24. Kiyak HA, West RA, Hohl T, et al. The psychological impact of orthognathic surgery: a 9-month follow-up. Am J Orthod. 1982;81(5):404 412.

25. Criteria for Orthognathic surgery. The American Association of Oral and Maxillofacial Surgeons. 2008

26. Cunningham SJ, Jilthorpe, MS, Hunt NP. Are pre-treatment psychological characteristics influenced by pre-surgical orthodontics? European Journal of Orthodontics. 2001;23(6):751-758.

27. Ireland AJ, Cunningham SJ, Petrie A, et al. An Index of Orthognathic Functional Treatment Need. J Orthod. 2014;41(2):77-83.

28. Modig M, Andersson L, Wardh I. Patient's perception of improvemen after orthognathic surgery: Pilot study. British Journal of Oral and Maxillofacial Surgery. 2005;44(1):24-27.

29. Pirklbaueret K, Russmueller G, Stiebellehner L, et al. Maxillomandibular Advancment for treatment of Obstructive Sleep Apnoea Syndrome: A systemic Review. J Oral Maxillofac Surg. 2011;69(6):e165-e176.

30. Sharma J, Sharma RD. IOTN-A tool to prioratize treatment need in children and plan dental health services. Oral Health and Dental Management. 2014;13:65-69. 